\title{
Integrated RMA with FMECA analysis using a graphical inteface: case study recycling iso- butane pump (CP2K)
}

\author{
El-Arkam Mechhoud ${ }^{(1)}$, Riad Bendib ${ }^{(2) .}$ Youcef Zennir ${ }^{(3)}$. \\ 1,2,3 University 20 August 1955, B.P.26 route El-Hadaiek Skikda-Algérie 21000 , Skikda, Algeria
}

*Corresponding Author: Email: elarkam_mechhoud@yahoo.fr /

el-arkam.mechhoud@univskikda.dz.

\begin{abstract}
The objective of our work is the implementation of a new approach for the dependability enhancement of a recycling iso-butane pump (Skikda CP2K petrochemical plant) based on automated dependability analysis system including the automation of the RMA (Reliability, Maintainability, Availability) study and FMECA (Failure Mode Effect and Criticality Analysis) method. After the system description, we present briefly the principle of the automated method. The system analysis in degraded mode is realized by using FMECA method. This automated analysis brings out the different failure modes, their causes and their consequences of each component. The result is risks minimization and reliability enhancement of the considered system.
\end{abstract}

Keywords: Risk analysis, FMECA, RMA, HDPE, Automated risk analysis

\section{INTRODUCTION}

The introduction section must contain theory related to the paper topic along with state of art study and well referenced citations. Make this section as informative as possible to justify the work and situate it with respect to the literature.

Due to the flammable, explosive and toxic nature of substances which are being handled in the oil-,gas- and petrochemical industries, installations within chemical plants have a high potential to cause substantial damages in terms of fatalities, serious injuries, property damages and environmental degradation. Petrochemical industry includes several sensitive area as storage area, reaction and conditioning area. The reaction is a very important and necessary operation in the petrochemical industry but it has many risks because intense temperature and pressure conditions and inherent congestion in process installations whereby process equipments are often situated in close proximity to one another, increases the probability of catastrophic accidents and increase their potential consequences. Among these accidents, the LNG (Liquefied Natural Gas) unit (GL1K-Skikda) explosion one that happened in 2004 in Skikda petrochemical plant, and where 27 people died while 112 others were severely injured.

Identification of major accident hazards relating to petrochemical activities and ensuring that the necessary measures have been taken to prevent and limit the consequences of such accident on man and the environment has become a necessity for companies. After the analysis study that applied to HDPE plant we extracted the different scenarios from the HAZOP study, the critical scenario is the clogging of the reactor due to the failure of the recycling $i$ butane pump [1]. Which causing the stop of production at least four months, for this reason we need to make an RMA study then the FMECA analysis of the recycling isobutane pump (CP2K Skikda plant).

In petrochemical industries, explosion risk must be analyzed for every component in the plant, and all tools must be used to minimize risk. Assessment of different kinds of risks by HAZOP \& FMECA methodology identifies human error and operational deviations. However, traditional risk assessment is consuming more time which often "makes mistakes". The automated dependability analysis tool that we propose in our paper would reduce the time and effort that furnish to risk analysis review, help operator to take good decision. In this task, we develop an interface which allows calculate the 
dependability indicators (RMA), identifying and localizing the causes of these failure modes.

This paper is structured in 5 main sections. The first one has made a brief introduction on the necessity of process hazard analysis. The following one briefly presents the FMECA study. The third one describes our approach. The fourth one applies the methodology proposed on the iso-butane pump and simulate the different indicators $R$, $M$ and $A$, and discus the different results that are obtained. The last section the conclusions are drawn and future works proposed.

\section{FAILURE MODE AND EFFECT ANALYSIS (FMEA)}

Failure Mode and Effects Analysis (FMEA) was developed during 1950s based on reliability of components. In this way, failure modes of all system components are recorded and then evaluated identifying potential hazards of the system [2].It is an approach that is often referred to as a "bottom up" approach, as it functions by means of the identification of a particular cause or failure mode within a system in a fashion that traces forward the logical sequence of this condition through the system to the final effects [3]. The main idea is to generate a risk priority number (RPN) for each failure mode. The first step in performing FMEA to analytical analysis is identification of potential failure modes. These failure modes are listed and then scored based on three aspects of the failure modes: occurrence (O), detection (D) and severity (S). For each of the failure modes, RPN values were calculated by:

$$
\mathrm{RPN}=\mathrm{O} \times \mathrm{D} \times \mathrm{S}
$$

The higher the risk number, the more serious the failure could be, and the more important it is that this failure mode be addressed [4]. FMECA (Failure Modes Effects and Criticality Analysis), is a method of criticality analysis by which each potential failure mode is ranked according to the combined influence of severity and probability of occurrence [5].

\section{AUTOMATED DEPENDABILITY APPROACH}

Our proposed tool for automated risk analysis is developed to enable a more global risk analysis, and dependability parameters estimation in the petrochemical plant. The software has been developed in graphical interface using Matlab as a coding tool.

The proposed concept involves the FMECA to analyze the risks and to calculate the dependability parameters by using the exponential rule to calculate reliability, maintainability and availability [6] to see the mean indicator of each component (as the pump, compressor, generator,...etc) in order to make a good, perfect analysis and make a safe system. Automated technique is used to support the decision-making process. In this framework our tool process involves creating two interlinked evaluation models. The first model is evaluated by the criticality matrix extracted from the FMECA analysis by the severity level implemented in the FMECA analysis and the second by the dependability indicators ( $R, M, A)$. Automated dependability analysis consists of four major elements (Figure 1): description and definition of the system, identification of hazards, RMA study and decision making.

- Description and definition of the system: The purpose of the first stage is to determine the system with all equipments and operating parameters by a decomposition of the global system into sub systems. We use a structural tree to identify all the equipments which build the system

- $\quad$ RMA study: The third stage helps to identify the state of the rotating machines. It includes a calculation of the dependability indicators $(R, M, A$, Mean Time Between Failure MTBF, Mean Time to repair MTTR, the failure rate $\lambda$ and repair rate $\mu$ ) assessed the failure probability and risk associated to a system situation, the information is provided to support the decision making process likely to be affected by the consequences of the possible accidents types [13]. But before this, we need to input the historic of each component [7]. We use the exponential rule to calculate and simulate the different parameters [8]. This stage helps the operator to prevent accidents and failures [9].

- Hazard identification: In this stage we proceed to hazard identification and localization. FMECA localize the problem by identifying all failure modes, their causes and their consequences in each sub-system elements. 
- Decision Making: After the analysis and evaluation of the risks by our tool, the user will be able to effectively localize the problem and to realize how much influence each evaluation criterion will have on the decision-making process and on the system safety.

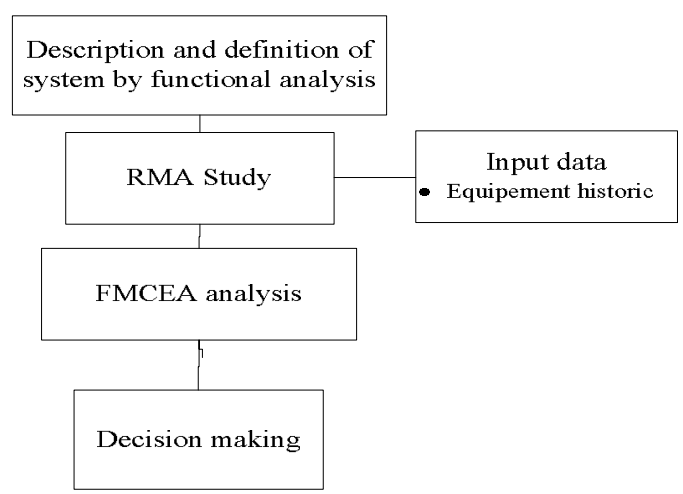

Fig.1 Flow chart of automated dependability analysis

\section{CASE STUDY}

\section{Process description}

The recycling iso-butane pump 670-172B will be considered as the case study. This system is at the High Density Polyethylene plant (CP2K) Skikda, Algeria. The CP2K plant is described by Mechhoud et al [10]. The recycling iso-butane pump $670-172 \mathrm{~B}$ is one the main equipment in the CP2K plant, because the recycling iso-butane has a principal role in the production of the HDPE, it uses as a solvent in the reaction, safety tool and washing system to protect the reactor. The recycling iso-butane supply pump in the dryer is centrifuged (figure.3) at high speed, operating at 12,290 rpm and operated by electric motor. The pump whose body is carbon steel and the internal are stainless steel, have design conditions for the pressure and temperature respectively of $69.3 \mathrm{~kg} / \mathrm{cm} 2 \mathrm{~g}$ and $70^{\circ} \mathrm{C}$. This pump provides a flow 32.6 $\mathrm{m} 3 / \mathrm{h}$, at discharge pressure $58.8 \mathrm{~kg} / \mathrm{cm} 2 \mathrm{~g}$ [11].

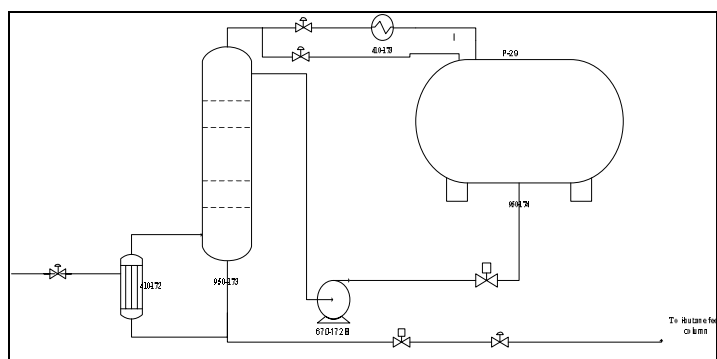

Fig. 2 Column of recycling iso-butane

\section{Application}

The effects of temperature, pressure and flow on the HDPE reactor are determined, and the operating condition for each case is selected. When we have a deviation in parameters, the HAZOP \& FMECA analysis is performed, but before the application of FMECA we need to calculate the dependability indicators to know the reliability of systems (rotating machines). The analysis will show the severity of the hazard level [12]. A human machine interface is constructed to automatically operate the HDPE process using RMA \& FMECA. This helps decreasing the hazards and increasing production as well as the reliability of the process.

Our analysis approach incorporates system modeling with functional analysis and safety analysis (FMECA). It can be divided into two parts. The first part is devoted a functional analysis to the decomposition of the system into three subsystems, as that the safety is done using it, each one having many equipments, while safety analysis is performed in the second part. A decomposition of the system is performed using graphical interface is developed under Matlab software. The safety analysis is preceded by the integration of RMA study and FMECA analysis. The analysis results are shown on the graphical interface. The RMA study is used to calculate all the dependability indicators such as reliability, maintenability and availability, while the FMECA analysis is applied to identify all failure modes, causes and consequences in each equipment.

Our developed graphical interface contains two modules: the functional analysis and the dysfunctional analysis [13].

Figure 3 shows the result of the FMECA analysis when the pressure increases in the reactor due to the iso-butane flow increasing. This flow increases because the i-butane pump failure, inadequate conditions of exploitation due to the malfunction of the instrumentations (sensors, control loop, control valve,..etc). In order to localize the problem (identify where is the problem exactly 
or in which part?) we use the RMA study to estimate the dependability parameters by clicking on the "RMA" button, then using the FMECA analysis by clicking on the "Pump" button (figure 3 ) to see the decomposition of the i-butane pump then the FMECA results (figure 4). The aim of applying the FMECA analysis after the HAZOP analysis is to identify and localize the problem in the pump, and to enhance and complete the necessary recommendations. Results of the two combined analysis will enable the user to effectively localize the problem and to realize how much influence each evaluation criterion will have on the decision-making process and on the system safety. The "elements" pop-up menu shows all the components in the system, selecting a component will show the results of the FMECA analysis concerning this component.

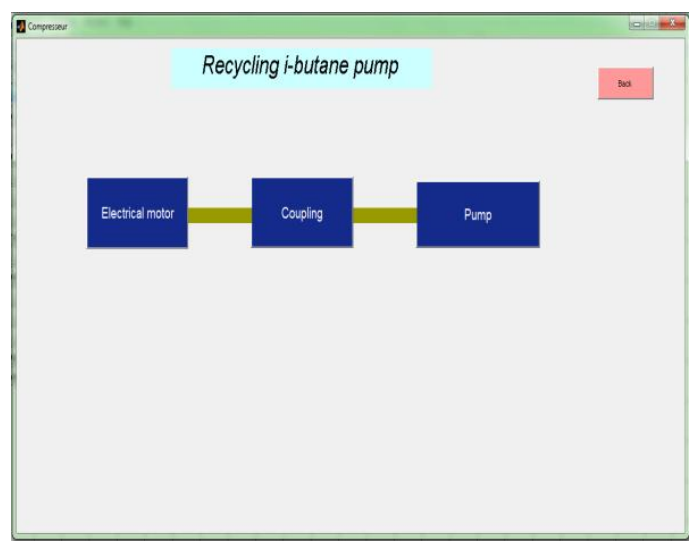

Fig.3 Graphical user interface- The recycling ibutane pump funtional analysis -

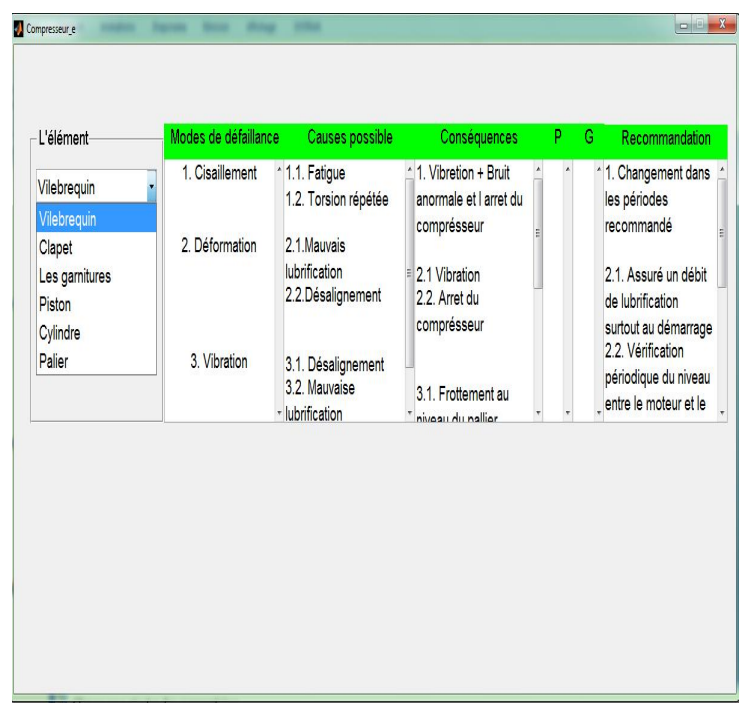

Fig.4 Graphical user interface -Dysfunctional analysis FMECA
Figure 5 shows the results of RMA assessment (the MTBF, MTTR, $\mu, \lambda, R, M, A$ ), it consists of four parts:

- Historic of equipment unit has the times of the system working and not working (function and failure)

- Specific times unit has TBF, TTR, MTBF, MTTR, Lamda and Mu

- RMA unit where set the RMA value, when we click on the "R" button we show the value of the reliability (Figure 5), when we click on the "M" button we show the maintainability value (Figure 6) and when we click on the "A" button we show the availability value (Figure 7).

- The axes RMA which trace the curves of reliability, maintainability or availability when we click on the button link of each one.

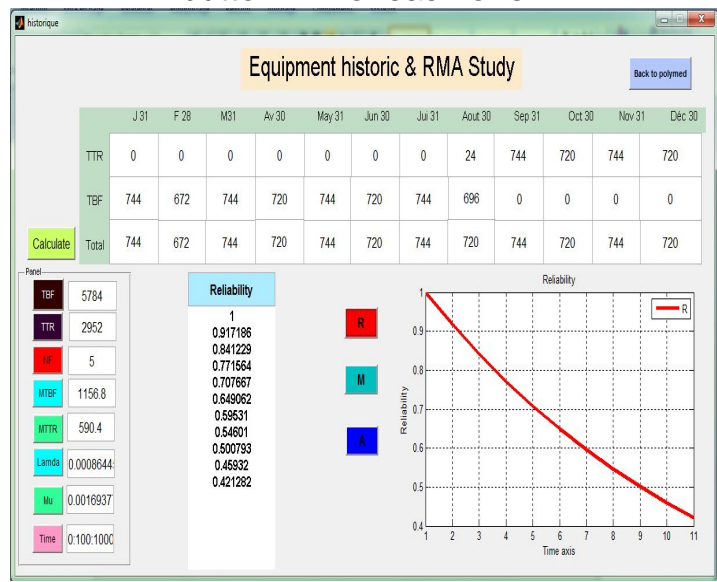

Fig.5 Calculate and draw of the I-B pump reliability

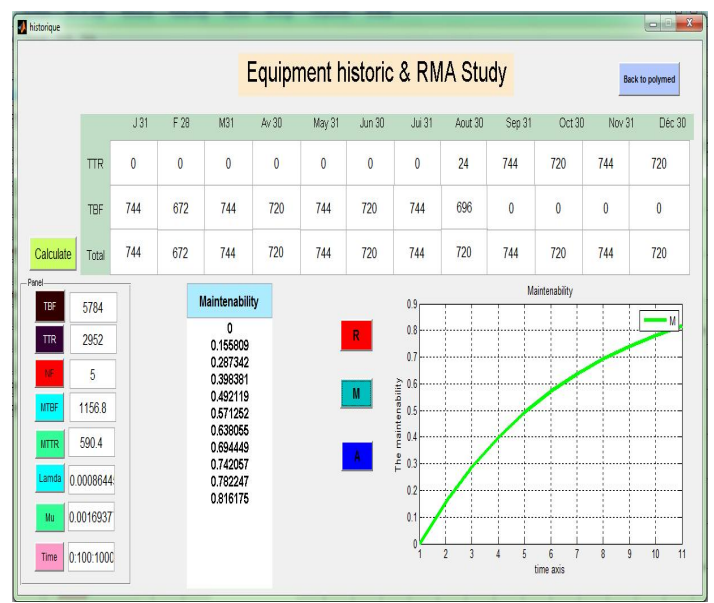

Fig.6 Calculate and draw of the I-B pump maintainability 


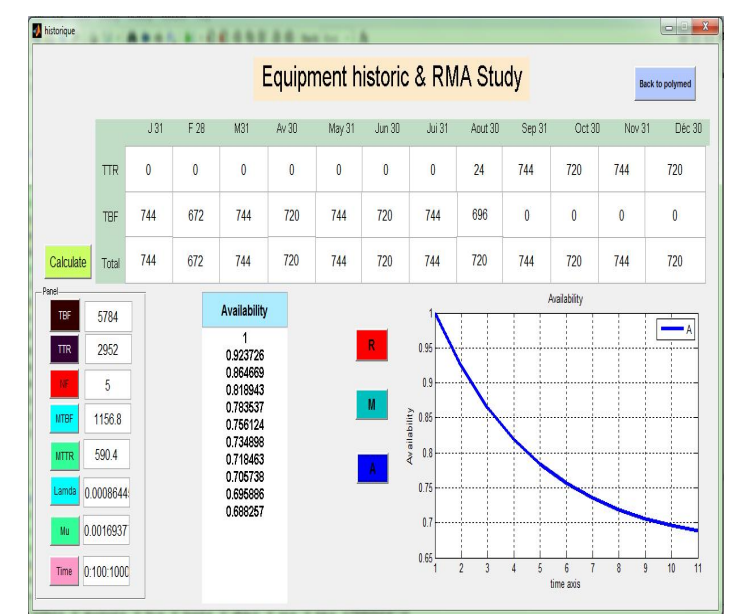

Fig.7 Calculate and draw of the I-B pump

\section{CONCLUSION}

In this paper, we developed an automated risk analysis approach. Built-in a graphical interface, the proposed method is dedicated to study the dependability of recycling isobutane pump systems in the Skikda CP2K plant. The system analysis in degraded mode is realized by FMECA analysis. This analysis brings out the different failure modes of the system, there possible causes, their consequences and preventive actions are identified and presented in the interface so as the user can easily operate them and make a correct actions.

The major contribution of our proposed approach is that beside the integration of RMA study and FMECA analysis which enhance risks assessments, it decreases the time consummation in hazard identification. The time spending is reduced compared with manual calculation thanks to the use of graphical interface which performs an online analysis. Results of the automated and integrated RMA \& FMECA analysis will enable the user to effectively localize the problem and to realize how much influence each evaluation criterion will have on the decision-making process and on the system safety. This approach of risks analysis and dependability study will be refined by the definition and propositions of the prevention means (safety barriers) for maximum decreases these risks and increasing the reliability and availability of the system's equipment.

\section{References}

[16] El Arkam Mechhoud, Mounira Rouainia, "Application de la méthode HAZOP su le réacteur PEHD", 9ème Congrés Francophon de Gènie des Procédés, Agadir- Marroc, 2014.

[17] Almannai B., Greenough R., Kay. J. A decision support tool based on QFD and FMEA for the selection of manufacturing automation technologies. Robotics and Computer-Integrated Manufacturing (2008). 24.vol 501-507.

[18] Mohamed N. LAKHOUA. (2009). Application of Analysis on aFunctional SCADA System of a Thermal Power Plant, Robotics and Computer-Integrated Manufacturing ., pp 11.

[19] McDermott R, Mikulak R, Beauregard, M. The basics of FMEA. Second edition USA: Productivity(2009).

[20] L. FROQUET, (2005). Contribution à l'analyse des risques : Proposition d'une méthode par scénarios et capitalisation de la connaissance". Thèse de doctorat de laboratoire d'automatique de Grenoble dans le cadre de l'école doctoral électronique, électrotechnique, automatique, télécommunication, signal, France,

[21] Smith. J Reliability, Maintainability and Risk, 7th edn. Elseiver Butterworth-Heinemann, Oxford, (2005). p.346.

[22] Barringer, H. Kotlya, P.E. Reliability Of Critical Turbo/Compressor Equipment, Fifth International Conference on Process Plant Reliability, Marriott Houston Westside Houston, Texas, October (1996). 2-4.

[23] Birollini, A. Reliability Engineering-Theory and Practice, Washington, D.C. (1999).

[24] Fernando V. Reliability Analysis of Centrifugal Pumps System Justifies Improvements in Gas Plant. Maintenance and Reliability Integrity Engineer, ABB Service, Argentina. (2005). p.9

[25] El-Arkam Mechhoud, Mounira Rouaïnia, Manuel Rdriguez. A new tool for risk analysis and assessment in petrochemical plants. Alexandria Engineering Journal (55), pp 2919-2931. 2016.

[26] Operation manual PHILIPS (CP2K)

[27] Bello, J-H. Impact of pump systems availability in the plant maintenance : Model development, A dissertation submitted to The University of Manchester for the degree of Master of Science in the Faculty of Engineering and Physical Sciences. p204. (2006).

[28] El. Mechhoud, M. Rouaïnia. AUTOMATED DEPENDABILITY ANALYSIS OF A HDPE REACTOR. 48 th ICCST-IEEE-. 13-16 October 2014, Rome- Italie. 